\title{
THIN FILM TRANSISTORS AND THIN FILM TRANSISTOR CIRCUITS
}

\author{
ANDRE VAN CALSTER \\ Laboratory of Electronics, State University, Sint Pietersnieuwstraat 41, B-9000 Gent, Belgium
}

In this paper the possible circuit applications of thin film transistors, made by shadow masks, are discussed. Special attention is paid to the basic parameters determining the circuit properties.

\section{INTRODUCTION}

The thin film transistor (TFT) is an insulated field-effect transistor made by a simple vacuum deposition process. Although a TFT may not be considered as a competitor of the Si MOSFET, the TFT technology shows some attractive qualities for special purpose circuits: the possibility of making large-area (macro) circuits with a built-in dielectric isolation, and the possibility of handling high voltages. A special feature of TFT technology is the possibility of obtaining a higher level of system integration. This is for instance the case in solid-state displays, where electronic and display functions are merged. ${ }^{1,2}$

Most of the developed TFT circuits are digital circuits. Less efforts are spent on analog circuits, probably due to the proven reliability of the Si technology in microelectronics. Nevertheless a higher integration level could also be possible in hybrids, using TFT technology. In order to check the feasibility of this goal, the characteristics of TFT test vehicles were investigated.

\section{TFT TECHNOLOGY}

The TFT structure used in our laboratory is shown in Figure 1. All depositions are made in one pumpdown of the vacuum deposition system, to minimize the device contamination during fabrication. By means of a mask changer Mo shadow masks are placed one by one in front of the substrate to delineate the device patterns. Devices with a channel length to width ratio of $30 \mu \mathrm{m} / 100 \mu \mathrm{m}$ have been made previously by metal masks. Prior to the source-drain deposition, the Corning 7059 substrate is coated with a $1000 \AA$ thick $\mathrm{Al}_{2} \mathrm{O}_{3}$ layer to prevent diffusion of impurities from the substrate.

The source and drain contacts are made by the successive deposition of $\mathrm{Au}$ and In by $\mathrm{E}$ gun. The $300 \AA$ thick semiconductor CdSe is evaporated from a heated Mo boat. The gate insulator $\mathrm{Al}_{2} \mathrm{O}_{3}$ is obtained by $\mathrm{E}$ gun evaporation, and the gate is an $800 \AA$ thick evaporated $\mathrm{Al}$ film. Afterwards the TFT is annealed in vacuum at $300^{\circ} \mathrm{C}$ for 3 hours. Finally the substrate is cut and gold wires are bonded to the TFT. In Figure 2 a TFT is shown mounted in a TO-5 package.

The stability of the device is mainly related to the insulator-semiconductor interface. ${ }^{3}$ $\mathrm{Al}_{2} \mathrm{O}_{3}$-CdSe seems to be one of the more stable combinations, with a sufficiently small number of slow surface states. ${ }^{4}$ The thin semiconductor film forms an n-channel, with a conductance determined by the applied gate voltage. The donor concentration is $510^{17}$ $\mathrm{cm}^{-3}$, and the electron mobility $\mu_{\mathrm{e}}$ equals $55 \mathrm{~cm}^{2} / \mathrm{Vsec}$. 


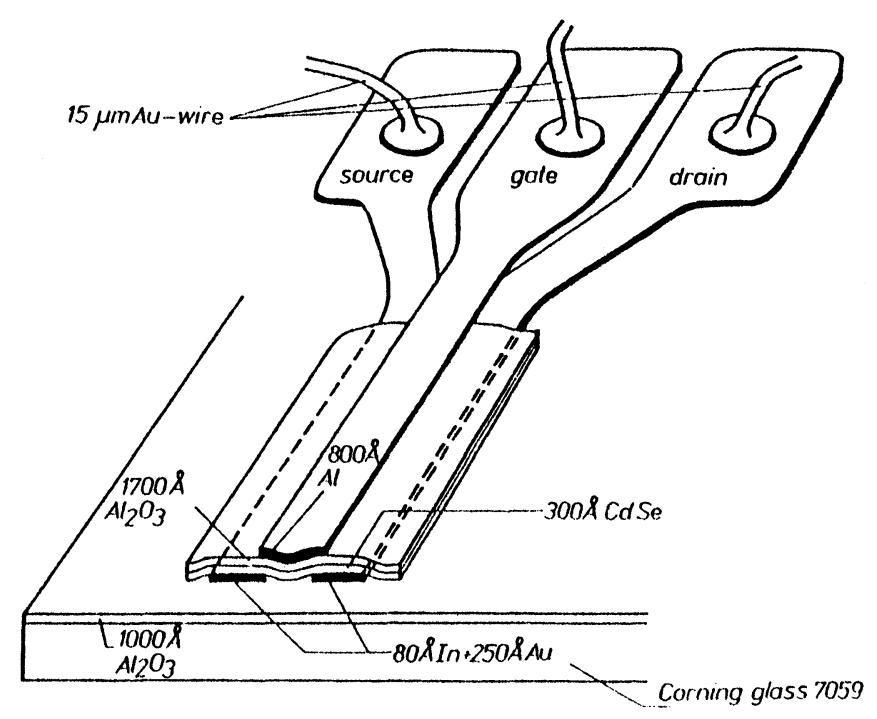

FIGURE 1 The thin film transistor (TFT) structure

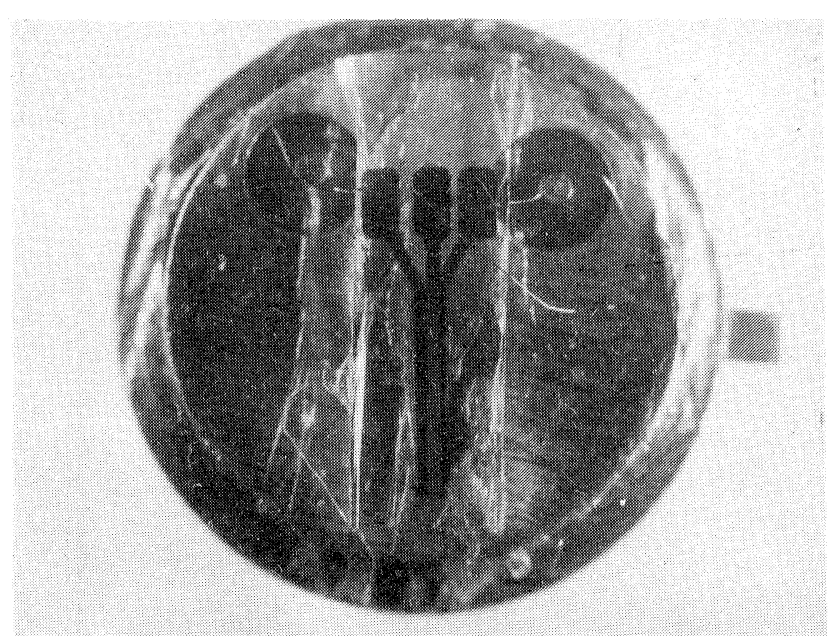

FIGURE 2 A TFT in a TO-5 package

\section{TFT CHARACTERISTICS}

In Figure 3 the small signal equivalent circuit is presented of a TFT biased at a gate voltage $V_{G}$ and a drain voltage $V_{D}$. Only for $V_{G}>0$ and $V_{D}>5^{v}$, the transconductance $\mathrm{g}_{\mathrm{m}}$ can be approximated by a linear relation as is predicted by simple theory. ${ }^{5}$

From Figure 3 it also follows that the turn-on voltage $V_{T}$ is negative, which means that the n-channel CdSe TFT behaves more like a depletion field-effect transistor. This means that in circuits one can use enhancement loads as well as depletion loads. When the TFT is biased at $\mathrm{V}_{\mathrm{G}} \approx 0^{\mathrm{v}}, \mathrm{g}_{\mathrm{m}}$ it can be fairly well approximated by a parabolic relation and the drain current $I_{D}$ by a third degree parabola. The data presented in Figure 3 are recorded for a TFT with a channel width to length ratio $S$ of 50 . 


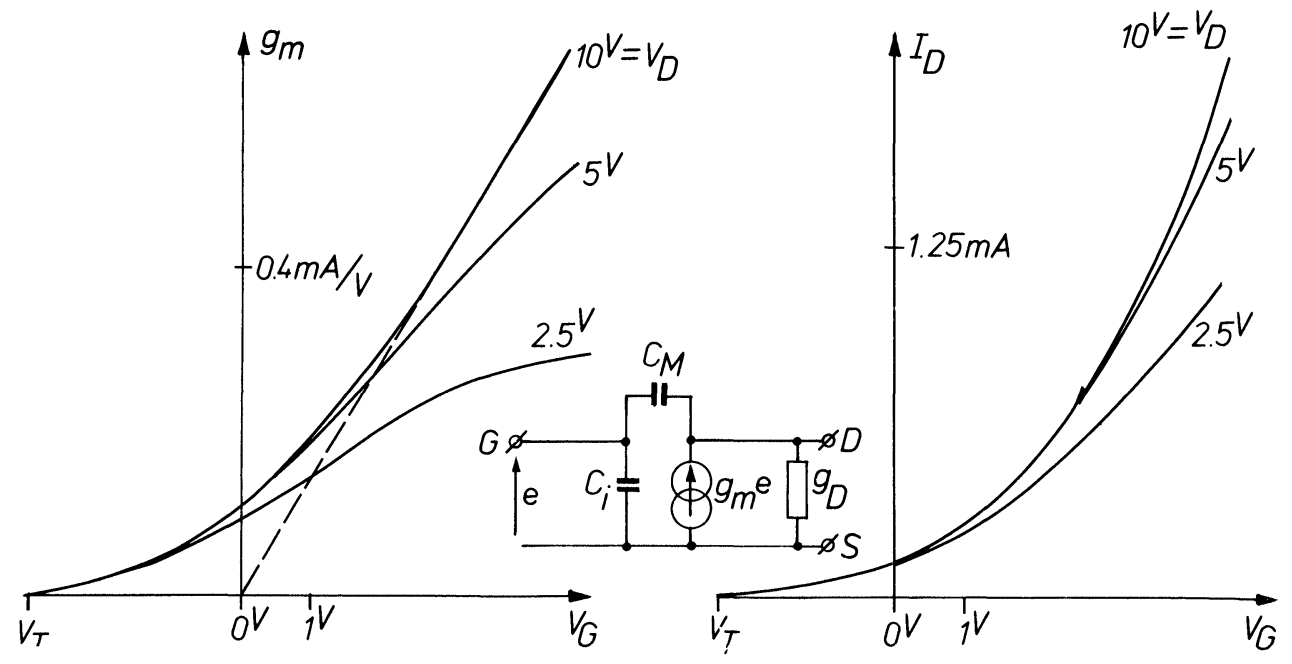

FIGURE $3 \quad g_{m}$ and $I_{D}$ versus $V_{G}$

In saturation $\left(V_{D}>V_{G}-V_{T}\right)$ the output conductance $g_{D}$ becomes $0.5 \mu S$. The input capacitance $C_{i}$ per unit area equals $5.210^{-8} \mathrm{~F} / \mathrm{cm}^{2}$, with a loss $\tan \delta=510^{-3}\left(\omega=10^{4}\right)$. With a $1700 \AA$ thick $\mathrm{Al}_{2} \mathrm{O}_{3}$ film the maximum $\mathrm{V}_{\mathrm{D}}$ is approximately $28^{\mathrm{v}}$. The frequency behaviour is determined by the unavoidable gate-drain electrode overlap. This causes the Miller capacitance $C_{M}$. The gain-landwidth $G \cdot B_{W}$ of the TFT presented in Figure 3 equals:

$\mathrm{G} \cdot \mathrm{B}_{\mathrm{W}}=\frac{\mathrm{L}}{\bar{\Delta}} \frac{1}{2 \pi \mathrm{T}_{\mathrm{r}}}$,

where $\mathrm{L}$ is the channel length, $\Delta$ the electrode overlap and $T_{r}$ the transit time of the TFT. $\mathrm{T}_{\mathrm{r}}$ is given by the expression:

$\mathrm{T}_{\mathrm{r}}=\frac{\mathrm{L}^{2}}{\mu_{\mathrm{e}}\left(\mathrm{V}_{\mathrm{G}}-\mathrm{V}_{\mathrm{T}}\right)}$,

and becomes $0.5 \mu \mathrm{sec}$ for $\mathrm{V}_{\mathrm{G}}=1^{\mathrm{v}}$ and $\mathrm{L}=60 \mu \mathrm{m}$.

An overlap of $10 \mu \mathrm{m}$ results in a G. $\mathrm{B}_{\mathrm{W}}=2 \mathrm{MHz}$. The equivalent circuit of Figure 3 is valid for frequencies smaller than $1 / 2 \pi T_{r}$.

In digital circuits the switching time of the device is of interest. In the absence of $\mathrm{C}_{M}$, the switching time is composed of a delay and risetime, both determined by $\mathrm{T}_{\mathrm{r}}$. The "on" and "off" state can be characterised by $g_{D}$. In the "on" state $V_{G}-V_{T}>V_{D}(\approx 0) g_{D}$ has a similar behaviour as $g_{m}$ at large $V_{D}, g_{D}$ increases linearly with $V_{G}$.

\section{TFT INVERTORS AND CIRCUITS}

The invertor stage may be considered as an elementary analog amplifier stage. Two types of load devices are possible: enhancement load (Figure 4a) and depletion load (Figure $4 \mathrm{~b}$ ). The enhancement load gave only an amplification of 2.5 , for a geometry ratio $S_{1} / S_{2}$ as large as 83 . With the depletion load is the fact that the maximum gain occurs at $\mathrm{V}_{\mathrm{G}}=0$, independent of $\mathrm{V}_{\mathrm{T}}$. Only the maximum attainable gain depends on $\mathrm{V}_{\mathrm{T}}$. 


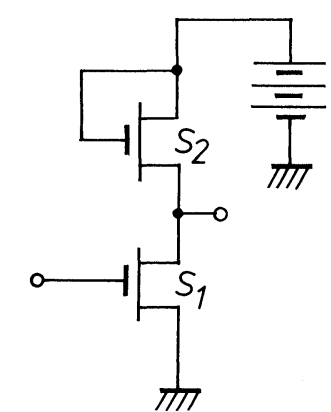

FIGURE 4a enhancement load

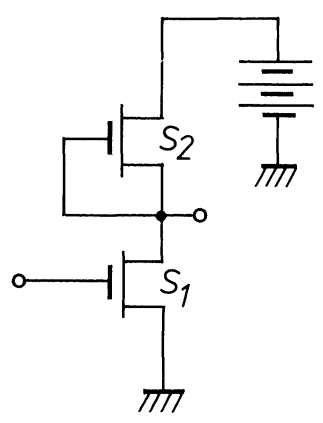

FIGURE $4 \mathrm{~b}$ depletion load

This is an interesting feature for designing digital circuits, where an invertor also may be considered as a basic circuit. For analog circuits, the circuit design should be process or $V_{T}$ insensitive, because $V_{T}$ differs from one batch to another. This means that the same design rules should be used as for nMOS. Different small circuits were tried out, such as multivibrators, Schmitt-triggers and analog followers. As an example a single stage voltage follower is shown in Figure 5, with measured input and output impendances respectively larger than $10 \mathrm{~m} \Omega$ and smaller than $10 \mathrm{~K} \Omega$.

We recently tackled the problem of the development of more complicated analog circuits, such as an operational amplifier.

\section{CONCLUSIONS}

From the above discussion it follows that TFT's can be made by a simple vacuum deposition process. By the use of the $\mathrm{Al}_{2} \mathrm{O}_{3}-\mathrm{CdSe}$ combination stable devices can be made. The use of mechanical masks still allows a suitable miniaturisation, but limits the frequency response due to the unavoidable electrode overlap. Nevertheless acceptable device characteristics can be obtained. TFT's with depletion loads show interesting circuit features

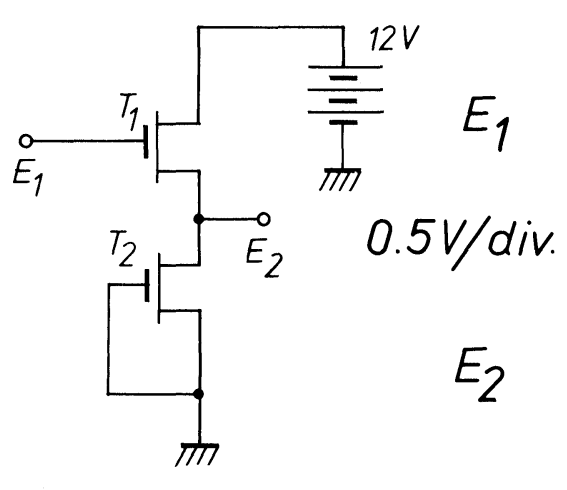

FIGURE 5 A voltage follower 
especially. This means that for special purpose circuits, digital as well as analog, where a higher integration level can be obtained, a TFT circuit solution should at least be considered.

\section{REFERENCES}

1. F. Luo and W. Hester, "Design and Fabrication of Large-Area Thin Film Transistor Matrix Circuits for Flat-Display Panels", IEEE Trans. ED 27 p. 223 (1980).

2. J. Erskine and P. Snopko, "A Thin-Film-Transistor-Controlled Liquid-Crystal Numeric Display", IEEE Trans. ED 26 p. 902 (1979).

3. A Van Calster and A. De Vos, "State of the Art in thin film transistor: a review of the used insulator-semiconductor combinations", ISHM Europe Gent Conference (1979).

4. A. Van Calster and $\mathrm{Li} \mathrm{Yu}$-Min, "An Investigation of the $\mathrm{Al}_{2} \mathrm{O}_{3}-\mathrm{CdSe}$ Interface in Accumulation", Appl. Phys, 23 p. 327 (1980).

5. A. Van Calster and H.J. Pauwels, "Theoretical influence of surface states and bulk traps on thin film transistor characteristics", Solid St. Electr. 18 p. 691 (1975). 

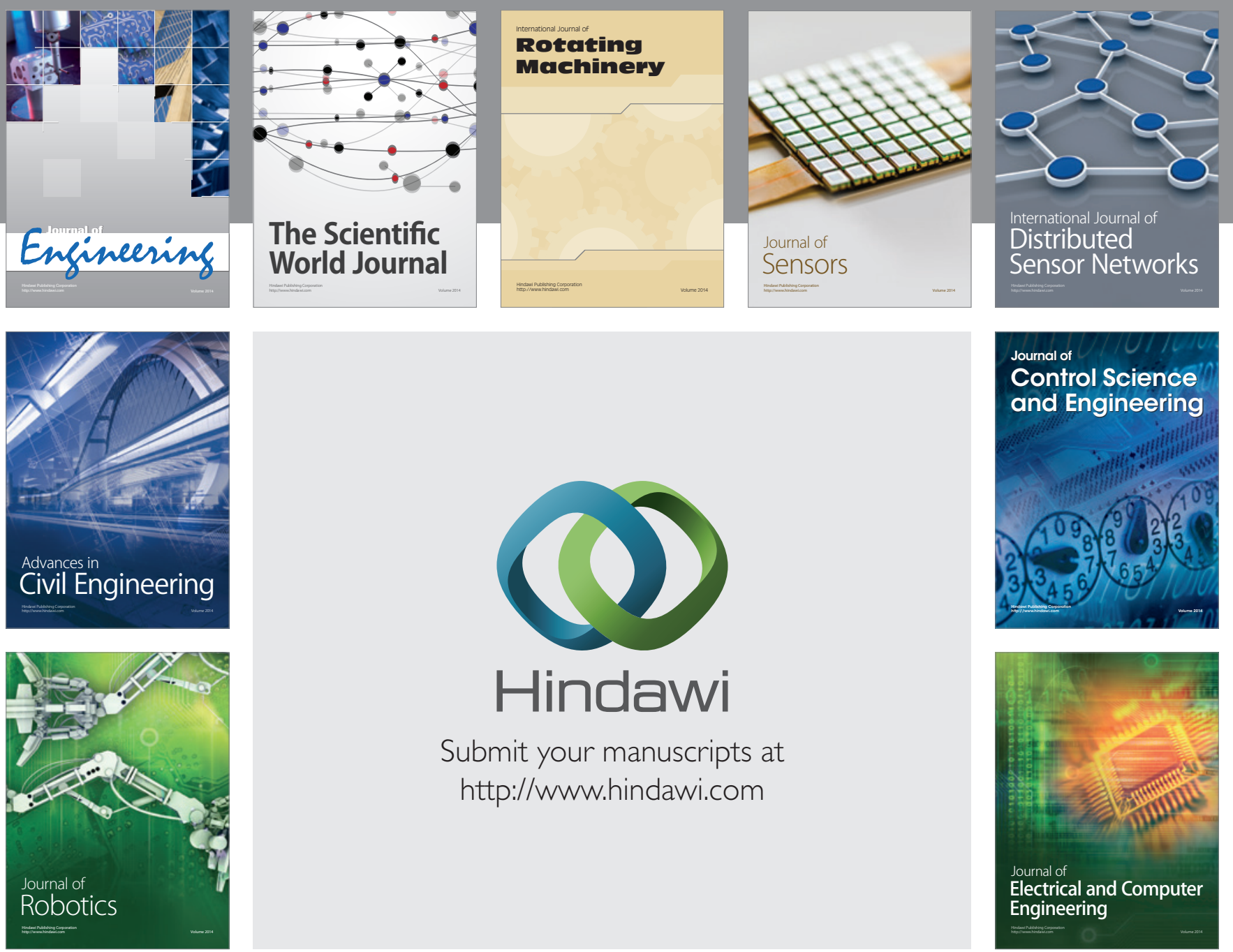

Submit your manuscripts at

http://www.hindawi.com
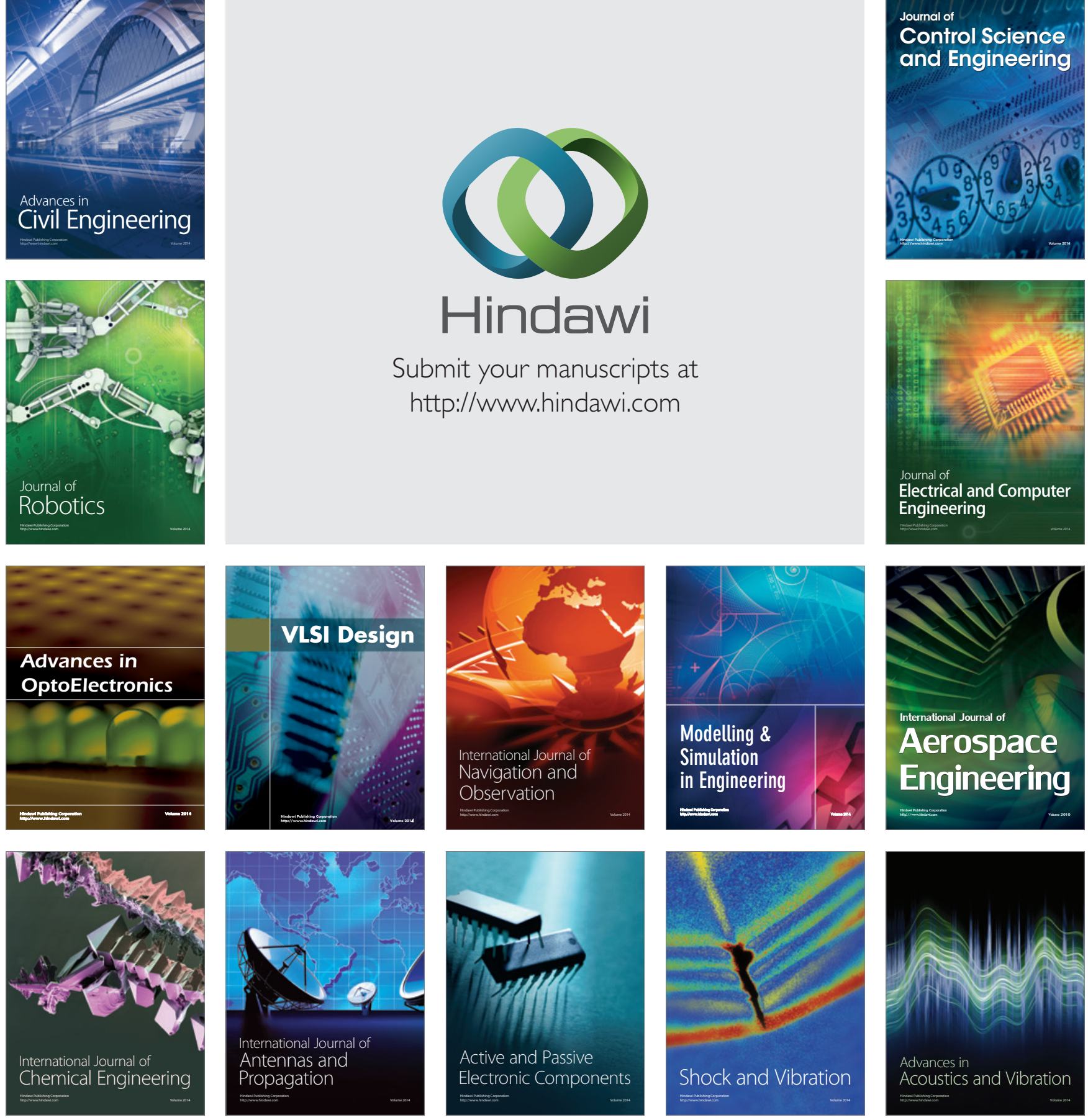